\title{
A influência da atividade física na qualidade de vida de idosas
}

\author{
Influence of physical activity on the quality of life of elderly women
}

\section{Michele Marinho da Silveiraa, Mirna Wetters Portuguez ${ }^{b}$}

a Fisioterapeuta. Professora na Faculdade Meridional (IMED). Pós-Doutoranda em Educação na Universidade Federal do Rio Grande do Sul, Brasil.

b Psicóloga. Doutora em Neurociência pela Universidade Federal de São Paulo. Professora na Faculdade de Medicina e no Programa de Pós-Graduação em Gerontologia Biomédica da Pontifícia Universidade Católica do Rio Grande do Sul (PUCRS). Pesquisadora do Instituto do Cérebro da PUCRS, Porto Alegre, RS, Brasil.

RESUMO

Objetivo: Verificar a influência da prática de atividade física na qualidade de vida de idosas.

Materiais e Métodos: Trata-se de um estudo transversal e descritivo com amostra de conveniência com 107 idosas de idade igual ou superior a sessenta anos pertencentes a grupos de convivência da região norte do Rio Grande do Sul, Brasil. Avaliaram-se os dados sociodemográficos, a atividade física e a qualidade de vida com escala WHOQOLBref, individualmente a cada idosa.

Resultados: A média de idade foi de $66,0 \pm 5,8$ anos, as idosas praticavam atividade física regularmente, em média, $222,6 \pm 61,1$ minutos semanais. O domínio físico apresentou o maior escore $(70,9 \pm 16,3)$ e o meio ambiente o menor $(67,2 \pm 13,9)$. Houve correlação entre o domínio físico frequência e tempo de atividade física, além de escolaridade, renda e autopercepção de saúde. O tempo de atividade física (beta $=0,188 ; p=0,026)$ e a frequência (beta $=0,191$; $p=0,023)$ se associaram com a qualidade de vida no domínio físico.

Conclusão: Observou-se que a maior frequência de atividade física está associada a maior pontuação no questionário WHOQOL-Bref.

Palavras-chave: envelhecimento; idosos; qualidade de vida; atividade física.

Objective: To analyze the influence of physical activity on the quality of life of older women.

Materials and Methods: It is a cross-sectional and descriptive study with a convenience sample of 107 elderly women aged 60 or older belonging to groups living in the northern region of Rio Grande do Sul, Brazil. Socio-demographic data, physical activity and quality of life using WHOQOL-Bref scale were evaluated individually for each elderly woman.

Results: A total of 107 older women were evaluated. The mean age was $66.0 \pm 5.8$ years, and the mean of regular physical activity

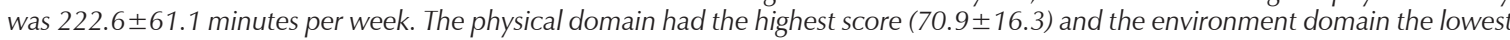
(67.2 \pm 13.9$)$. There was a strong correlation between the physical domain frequency and time of physical activity, in addition to schooling, income and self-perception of health. The physical activity time (beta $=0.188 ; p=0.026)$ and the frequency (beta $=0.191$; $p=0.023$ ) were associated with the quality of life in the physical domain.

Conclusion: It was observed that a higher frequency of physical activity is associated with a higher score on the WHOQOL-Bref questionnaire.

Keywords: aging; elderly; quality of life; physical activity. 


\section{INTRODUÇÃO}

O processo de envelhecimento tem se apresentado como fenômeno comum em países economicamente estáveis e em desenvolvimento ${ }^{1,2}$. O aumento do tempo de vida está relacionado à senilidade com níveis de saúde e condições de vida satisfatórias na perspectiva do envelhecimento com qualidade de vida. O avanço da idade é, geralmente, acompanhado pelo declínio das funções biológicas, físicas/ motoras e cognitivas, com maior incidência e agravos à saúde³ , colocando os idosos numa condição de vulnerabilidade ${ }^{4}$.

Em vista disso, na tentativa de minimizar o impacto do processo de envelhecimento na vida diária dos idosos, surgem grupos de convivência que proporcionam programas de envelhecimento ativo, alternativa estimulada em todo o Brasil $^{5}$. De maneira geral, os idosos buscam nesses grupos melhoria física e mental por meio de exercícios físicos e atividades recreativas.

A prática de atividade física é uma forma independente para reduzir e/ou prevenir uma série de declínios funcionais associados ao envelhecimento ${ }^{6,7}$. Assim, os principais benefícios de um comportamento ativo do idoso podem ser classificados, basicamente, nas esferas biológica, psicológica e social, dentre os quais destacam-se ${ }^{8}$ : a) aumento/manutenção da capacidade aeróbia; b) aumento/manutenção da massa muscular e redução do risco de sarcopenia; c) redução da taxa de mortalidade total; d) prevenção de doenças coronarianas; e) prevenção/controle da diabetes tipo II e hipertensão arterial; f) redução da ocorrência de acidente vascular cerebral e demência; g) prevenção primária do câncer de mama e cólon; h) diminuição da ansiedade e do estresse; i) melhora do estado de humor e da qualidade de vida.

A prática regular de atividade física proporciona melhoria das condições de saúde no processo de envelhecimento9 ${ }^{9}$. A atividade física, além dos benefícios orgânicos nos aspectos metabólico, cardiorrespiratório, muscular e articular, contribui significativamente para promoção da independência, consequentemente, de uma satisfatória qualidade de vida ${ }^{10,11}$. Pesquisadores revelam que o nível de atividade física pode interferir na capacidade funcional, influenciando na saúde e qualidade de vida dos idosos ${ }^{12}$.

Para Mazo et al..$^{13}$ mulheres idosas que praticam atividade física e são mais ativas ( $\geq 150 \mathrm{~min} / \mathrm{sem}$ ) têm médias mais elevadas nos domínios psicológicos e físicos de qualidade de vida e as que apresentaram um pior resultado no domínio físico da qualidade de vida têm um risco três vezes maior de serem menos ativas ( $<150 \mathrm{~min} / \mathrm{sem})$ fisicamente, demonstrando que esses resultados sugerem que a atividade física desempenha um papel importante na qualidade de vida das mulheres idosas. Já para outros pesquisadores ${ }^{14} \mathrm{a}$ qualidade de vida não apresentou associações com melhores níveis quando comparados aos grupos mais ativos e menos ativos de idosas.

Em vista disso, o objetivo deste estudo foi verificar a influência da prática de atividade física na qualidade de vida de idosas participantes de grupos de convivência. Os objetivos específicos foram: verificar as características da atividade física; analisar os domínios de qualidade de vida e, por fim, correlacionar atividade física com qualidade de vida.

\section{MATERIAIS E MÉTODOS}

Este estudo transversal descritivo contou com indivíduos recrutados por meio da técnica de amostragem por conveniência. A amostra foi composta por 107 idosas de dois grupos de convivência no município de Passo Fundo-RS (Centro Regional de Estudos e Atividades a Terceira Idade e Coordenadoria de Atenção ao Idoso), grupos estes que são predominantemente compostos por mulheres.

Para participar do estudo, os sujeitos deveriam estar inseridos nos grupos de convivência e ter idade igual ou superior a sessenta anos, ser do sexo feminino e dar o seu consentimento para participar do estudo assinando termo de consentimento livre e esclarecido TCLE. Como critérios de exclusão, definiram-se os indivíduos que não tinham capacidade de comunicação verbal ou escrita e de compreensão do TCLE e dos questionários. Todas as entrevistadas foram incluídas no estudo sem apresentar motivos para sua exclusão.

Como instrumentos, um questionário sociodemográfico e de atividade física elaborado pelos próprios autores, que incluiu as variáveis idade; escolaridade; renda; estado civil; frequência semanal de atividade física; tempo de cada atividade física realizada; tipo de atividade física e percepção de saúde. Também foi utilizada a escala WHOQOL-Bref que é um instrumento que avalia quatro domínios de qualidade de vida (físico, psicológico, relações sociais e meio ambiente), incluindo questões de avaliação global de qualidade de vida que geram um escore global, chamado "qualidade de vida geral". Este instrumento foi desenvolvido a partir da WHOQOL-100 e validado para o português por Fleck et al. ${ }^{15}$, consta de 26 questões, duas gerais sobre qualidade de vida e 24 outras que representam quatro domínios. Não tem ponto de corte, quanto maior a pontuação, que varia de 0 a 100, melhor a qualidade de vida.

O projeto foi examinado e aprovado pela Comissão Científica do Instituto de Geriatria e Gerontologia da Pontifícia Universidade Católica do Rio Grande do Sul (PUCRS) e também pelo Comitê de Ética da mesma universidade em Porto Alegre-RS, Brasil, parecer no 241.858 . Após a aprovação, foram realizados contatos com os grupos 
de idosos, procedendo-se, assim, à inclusão dos participantes na amostra. Todos os sujeitos que concordaram em participar foram entrevistados individualmente pelo mesmo entrevistador e cada um preencheu o TCLE e respondeu a informações sociodemográficas e de qualidade de vida.

Os dados foram armazenados no MS Excel 2010 para Windows, e posteriormente analisados pelo programa Statistical Package for the Social Sciences (SPSS) para Windows, versão 21.0. A normalidade foi avaliada pelo teste de Kolmogorov-Smirnov. As variáveis quantitativas foram descritas por média e desvio padrão e as categóricas, por frequências absolutas e relativas. Para comparar as médias entre os quatro domínios do WHOQOL-Bref, foi aplicada a análise de variância (Anova). Para análise de correlação o teste de Pearson foi utilizado para dados paramétricos e o de Spearman para dados não-paramétricos. Foram considerados como estatisticamente significativos testes com valor de probabilidade $<0,05$. Para controle de fatores confundidores na relação entre atividade física e domínio físico do WHOQOL-Bref, foi utilizada a análise de regressão linear múltipla. O coeficiente de correlação padronizado ( $\beta$ ) foi utilizado para avaliar o grau da associação entre as variáveis. O nível de significância adotado foi de 5\% $(p<0,05)$.

\section{RESULTADOS}

A média de idade das idosas entrevistadas foi de $66,0 \pm 5,8$ anos e os de tipos de medicamentos administrados por dia foi de $3,32 \pm 2,37$. Apenas dez $(9,3 \%)$ idosas não faziam uso de medicamentos, sendo que $14(13,1 \%)$ faziam uso de um tipo de medicamento, 22 (20,6\%) de dois tipos, vinte $(18,7 \%)$ de três tipos, $11(10,3 \%)$ de quatro e entre cinco e dez tipos de medicamentos trinta (28\%) idosas. $\mathrm{Na}$ Tabela 1 observam-se as características sociodemográficas das idosas e sua autopercepção de saúde.

Entre os tipos de atividades físicas realizadas são alongamento e fortalecimento muscular (oficina de $1 \mathrm{~h}$ e $30 \mathrm{~min})$, alongamento muscular (1h), hidroginástica (1h), dança (1h e 30min) e yoga (1h). A média de minutos de atividade física por semana entre as idosas foi de 222,6 $\pm 61,1$ observados na Tabela 2.

Na Tabela 3 são apresentadas as médias dos domínios de qualidade de vida. Houve diferença significativa entre as médias dos domínios do WHOQOL-BREF $(p=0,011)$ e os idosos pontuaram menos no meio ambiente quando comparado com a pontuação no domínio físico.

As correlações entre os dados de atividade física e os dados sociodemográficos com os domínios da qualidade de vida estão apresentados na Tabela 4. Houve correlação positiva estatisticamente significativa entre a frequência de atividade física e o tempo da atividade (minutos por semana)
Tabela 1. Características sociodemográficas e autopercepção de saúde da amostra $(n=107)$

\begin{tabular}{lcc}
\multicolumn{1}{c}{ Variáveis } & $\mathbf{n}$ & $\%$ \\
Faixa etária (anos) & & \\
$60-69$ & 79 & 73,8 \\
$70-79$ & 26 & 24,3 \\
$80-89$ & 2 & 1,9 \\
Estado Civil & & \\
$\quad$ Solteira & 10 & 9,3 \\
Casada & 47 & 43,9 \\
Separada/Divorciada & 16 & 15,0 \\
Viúva & 34 & 31,8 \\
Nível de escolaridade (anos) & & \\
4 & 21 & 19,6 \\
8 & 22 & 20,6 \\
11 & 31 & 29,0 \\
12 ou mais & 33 & 30,8 \\
Renda (salário mínimo) & & \\
1-2 & & \\
3-4 & 64 & 59,8 \\
5-6 & 26 & 24,3 \\
7 ou mais & 10 & 9,3 \\
Autopercepção de saúde & 7 & 6,5 \\
Ótima & & \\
Boa & & 12,1 \\
Regular & & 49,5 \\
Ruim & 13 & 37,4 \\
\hline
\end{tabular}

Tabela 2. Características da atividade física da amostra $(n=107)$.

\begin{tabular}{lcc}
\hline \multicolumn{1}{c}{ Variáveis } & $\mathbf{n}$ & $\%$ \\
Frequência (vezes/semana) & & \\
$2 \mathrm{x}$ & 65 & 60,7 \\
$3 \mathrm{x}$ & 16 & 15,0 \\
$4 \mathrm{x}$ & 21 & 19,6 \\
$5 \mathrm{x}$ & 4 & 3,7 \\
$6 \mathrm{x}$ & 1 & 0,9 \\
Tipos diferentes de atividade física & & \\
1 & 63 & 58,9 \\
2 & 19 & 17,8 \\
3 & 21 & 19,6 \\
4 & 3 & 2,8 \\
5 & 1 & 0,9 \\
Minutos/semana & & \\
180 & & \\
240 & 64 & 59,8 \\
300 & 18 & 16,8 \\
360 & 20 & 18,7 \\
420 & 2 & 1,9 \\
\hline
\end{tabular}

Tabela 3. Escores de qualidade de vida - WHOQOL-Bref $(n=107)$.

\begin{tabular}{lc}
\hline \multicolumn{1}{c}{ Domínios } & Média \pm desvio padrão \\
\hline Físico & $70,9 \pm 16,3^{\mathrm{b}}$ \\
Psicológico & $69,5 \pm 13,1^{\mathrm{ab}}$ \\
Relações sociais & $70,2 \pm 18,6^{\mathrm{ab}}$ \\
Meio ambiente & $67,2 \pm 13,9^{\mathrm{a}}$ \\
Global & $70,8 \pm 16,6$ \\
\hline
\end{tabular}

a,bLetras iguais não diferem pelo teste de Bonferroni a 5\% de significância. 
Tabela 4. Correlações dos dados de atividade física e sociodemográficos com os domínios da qualidade de vida.

\begin{tabular}{lccccccc}
\hline & Frequência AF & Tipo de AF & Minutos/semana & Idade & Escolaridade & Renda & AP de saúde \\
Físico & $0,191^{*}$ & 0,178 & $0,208^{*}$ & 0,021 & $0,231^{*}$ & $0,259^{* *}$ & $-0,498^{* *}$ \\
Psicológico & 0,085 & 0,098 & 0,119 & 0,173 & 0,028 & 0,079 & $-0,330^{* *}$ \\
Relações sociais & 0,170 & 0,162 & 0,188 & 0,163 & $-0,048$ & $-0,024$ & $-0,170$ \\
Meio ambiente & 0,171 & 0,138 & $0,190^{*}$ & 0,171 & 0,089 & $0,271^{* *}$ & $-0,326^{* *}$ \\
\hline
\end{tabular}

*Correlação significativa ao nível de 0,$05 ;{ }^{* *}$ Correlação significativa ao nível de 0,01. AF: atividade física; AP: autopercepção.

somente com o domínio físico do WHOQOL-Bref, ou seja, quanto mais frequente e maior tempo de atividade física, melhor o escore nesse domínio.

Em relação às demais variáveis, o nível de escolaridade se correlacionou positivamente também com o domínio físico, demonstrando que as idosas com maior escolaridade têm pontuação mais elevada nesse domínio. A renda se correlacionou positivamente com os domínios físico e meio ambiente, demonstrando que quanto maior a renda, melhor a qualidade de vida nesses domínios. A autopercepção de saúde se correlacionou positivamente com praticamente todos os domínios, exceto as relações sociais. Quanto pior a autopercepção de saúde dos idosos, pior a avaliação de sua qualidade de vida (Tabela 4). Os escores de qualidade de vida não se correlacionaram significativamente com estado civil e apenas o domínio físico se correlacionou ao uso de medicamentos diários $(r=-0,265)$, demonstrando que quanto maior o número de medicamentos, menor a qualidade de vida nesse domínio.

Em relação ao domínio físico, quando o tempo de atividade física é ajustado para escolaridade, renda, auto percepção de saúde e número de medicações por dia, a associação permanece estatisticamente significativa após o ajuste (beta $=0,188 ; p=0,026$ ), demonstrando que o tempo de atividade física é um fator independentemente associado com a qualidade de vida no domínio físico. A frequência de atividade física também permanece associada com a qualidade de vida no domínio físico após o ajuste pelos mesmos fatores (beta $=0,191 ; p=0,023$ ) vistos na Tabela 5 .

Tabela 5. Análise de regressão linear multivariada para avaliar fatores independentemente associados com o domínio físico do WHOQOL-Bref.

\begin{tabular}{lccc}
\hline \multicolumn{1}{c}{ Variáveis } & B (IC 95\%) & Beta & $\boldsymbol{p}$ \\
AF minutos/semana & $0,05(0,01$ a 0,09$)$ & 0,188 & $\mathbf{0 , 0 2 6}$ \\
AF vezes/semana & $3,18(0,44$ a 5,92$)$ & 0,191 & $\mathbf{0 , 0 2 3}$ \\
Nível de escolaridade & $0,31(-2,50$ a 3,12$)$ & 0,021 & 0,829 \\
Autopercepção de saúde & $-8,58(-13,2$ a $-3,95)$ & $-0,359$ & $<\mathbf{0 , 0 0 1}$ \\
Renda & $1,60(-1,35$ a 4,55$)$ & 0,103 & 0,285 \\
N $^{\circ}$ de medicações por dia & $-1,03(-2,18$ a 0,12$)$ & $-0,150$ & 0,079 \\
\hline
\end{tabular}

B: coeficiente de regressão (efeito na qualidade de vida no domínio físico a cada aumento de uma unidade do fator); IC 95\%: intervalo com 95\% de confiança; Beta: coeficiente de regressão padronizado (desconsidera a unidade da variável, é como se fosse um coeficiente de correlação ajustado).

\section{DISCUSSÃO}

Inicialmente, procurou-se caracterizar o perfil das idosas participantes do estudo que apresentavam faixa etária prevalente entre 60-69 anos, o que corrobora com a média da população idosa verificada pelo Instituto Brasileiro de Geografia e Estatística ${ }^{16}$, onde no ano de 2010 essa faixa etária era a mais encontrada. Além disso, a maioria das idosas deste estudo era casada, com bom nível de escolaridade e renda razoável. De acordo com diversas pesquisas, a maior parte dos frequentadores de grupos de convivência é constituída de mulheres casadas ${ }^{11,17}$, com faixa etária entre 60-69 anos, bom nível de escolaridade ${ }^{18}$ e renda entre um e dois salários mínimos ${ }^{19}$.

A autopercepção de saúde nesta pesquisa se associou positivamente com, praticamente, todos os domínios, exceto com as relações sociais, demonstrando que quanto pior a autopercepção de saúde dos idosos, pior a avaliação de sua qualidade de vida. A autopercepção de saúde da maioria das idosas desta pesquisa foi considerada boa, corroborando com outros estudos ${ }^{20,21}$, entretanto discordando com alguns pesquisadores que revelaram que os idosos de grupos de convivência relataram sua autopercepção de saúde regular ${ }^{22}$.

Pesquisas revelam que mulheres idosas que praticam atividade física apresentam uma qualidade de vida satisfatóriaa ${ }^{14,23}$. Nesta pesquisa, todas as idosas eram fisicamente ativas, revelando que a prática regular de atividade física influenciou beneficamente a qualidade de vida das idosas. Outros estudos, que compararam a qualidade de vida de idosos que praticavam atividade física e idosos sedentários, revelaram que um estilo de vida ativo pelos praticantes de atividade física resultou em melhor qualidade de vida ${ }^{23,24}$. Até em instituições de longa permanência, idosos mais jovens, com maior escolaridade, que realizavam atividade física e de lazer, apresentaram, em média, melhor percepção na qualidade de vida, demonstrando o quanto a atividade física é importante para a qualidade de vida de quem envelhece ${ }^{25}$. Em um estudo com 863 idosos, no sul do Brasil, foi observado que quanto mais ativo é o indivíduo, melhor é a sua qualidade de vida, incluindo não apenas as questões 
relacionadas à saúde, mas também aos aspectos psicológicos e $\operatorname{cognitivos}^{26}$.

O domínio físico da qualidade de vida apresentou a maior média, e esse representa dor, desconforto, relação com a dependência de medicação/tratamentos, energia e fadiga, mobilidade, sono e repouso, atividades da vida cotidiana e capacidade de trabalho, revelando que essas idosas apresentam boa disposição, mobilidade e capacidade funcional corroborando com alguns autores ${ }^{19,27}$. Os efeitos da prática de atividade física correlacionada ao domínio físico demonstram uma correlação positiva e significativa entre ambos, corroborando com outros pesquisadores ${ }^{28}$.

Por sua vez, o domínio psicológico envolve os sentimentos negativos e positivos, como o aproveitar a vida, o otimismo em relação ao futuro, a preocupação com condições de doença ou a ausência desta, espiritualidade, crenças pessoais, memória e concentração, imagem corporal, aparência e autoestima. Para as participantes, esse domínio comprova os sentimentos positivos em relação ao aproveitar a vida em concordância com alguns pesquisadores ${ }^{17,29}$.

O domínio das relações sociais se refere às relações pessoais, atividade sexual e suporte social. Alguns autores argumentam que, talvez, o ato sexual em si seja menos importante nessa faixa etária do que a possibilidade de intimidade, e que a imagem corporal/aparência não seja tão valorizada para os idosos quanto para pessoas mais jovens ${ }^{30}$. Entretanto, ao contrário desses autores, as participantes desta pesquisa demonstraram que as relações sociais influenciam de maneira positiva as suas vidas.

O domínio meio ambiente se refere à segurança física e proteção, ambiente físico, recursos financeiros, oportunidade de adquirir novas informações e habilidades, participação em oportunidade de lazer, ambiente do lar, cuidados de saúde e sociais, como disponibilidade, qualidade e transporte, vindo a comprovar pelas condições socioeconômicas das participantes visualizadas na Tabela 1. Entretanto foi o domínio que apresentou menor escore, corroborando os achados de Tavares \& Dias ${ }^{31}$.

Pesquisadores avaliaram o nível de atividade física de idosos participantes de grupos de convivência e verificaram que eles são mais ativos fisicamente e as mulheres idosas são mais ativas que os homens idosos ${ }^{32}$. Além disso, idosas participantes de grupos de convivência que são fisicamente ativas têm melhor qualidade de vida ${ }^{24}$.

Acredita-se que se está contribuindo no acréscimo de dados empíricos à literatura brasileira sobre a influência da prática de atividade física na qualidade de vida de idosas. Diante das informações observadas, remete-se à necessidade de sensibilizar os profissionais de saúde que lidam com idosos para os benefícios da prática de atividades físicas, no sentido de implementá-las sistematicamente junto a essa população. Também, sugere-se que seja incentivada a criação de novos grupos e programas voltados a atividade física, que proporciona tantos benefícios aos que envelhecem.

Como limitações encontradas neste estudo, a definição da amostra pode ser considerada um fator limitante visto que não foi aleatória. Além disso, a amostra deste trabalho engloba apenas idosas participantes de grupos de convivência, por isso, não podemos generalizar os resultados obtidos a outras idosas que não pertencem a esses grupos.

Por fim, podemos concluir que a prática de atividade física de idosas participantes de grupos de convivência contribuiu para uma boa qualidade de vida. Observou-se que quanto maior a frequência e o tempo de atividade física, ou seja, quanto mais ativas eram as idosas, melhor se apresentou a qualidade de vida. Sugerem-se novos estudos que abordem a mesma temática, porém com metodologias diversificadas visando qualificar os resultados.

\section{REFERÊNCIAS}

1. Duarte EC, Barreto SM. Transição demográfica e epidemiológica: a Epidemiologia e Serviços de Saúde revisita e atualiza o tema. Epidemiol Serv Saúde. 2012;21(4):529-32. https://doi.org/10.5123/ S1679-49742012000400001

2. Wójcicki TR, Roberts SA, Learmonth YC, Hubbard EA, KinnettHopkins D, Motl RW, McAuley E. Improving physical functional and quality of life in older adults with multiple sclerosis via a DVD-delivered exercise intervention: a study protocol. BMJ Open. 2014;4(12):e006250. https://doi.org/10.1136/ bmjopen-2014-006250

3. Hayter AKM, Jeffery R, Sharma C, Prost A, Kinra S. Community perceptions of health and chronic disease in South Indian rural transitional communities: a qualitative study. Glob Health Action. 2015;8:25946. https://doi.org/10.3402/gha.v8.25946.

4. Wong F, Stevens D, O'Connor-Duffany K, Siegel K, Gao Y; Community Interventions for Health $(\mathrm{ClH})$ collaboration. Community Health Environment Scan Survey (CHESS): a novel tool that captures the impact of the built environment on lifestyle factors. Glob Health Action. 2011;4:5276. https://doi.org/10.3402/gha.v4i0.5276

5. Wichmann FMA, Couto AN, Areosa SVC, Monta-és MCM. Grupos de convivência como suporte ao idoso na melhoria da saúde. Rev Bras Geriatr Gerontol. 2013;16(4):821-32. https://doi.org/10.1590/ S1809-98232013000400016

6. Gonçalves MM. Atividade física e funcionalidade do idoso. Motriz: Rev Educ Fís. 2010;16(4):1024-32. https://doi.org/10.5016/19806574.2010v16n4p1024

7. Rodrigues PCS, Barreiros JMP, Vasconcelos MOF, Carneiro SRM. Efeito da prática regular de atividade física no desempenho motor em idosos. Rev Bras Educ Fís Esporte. 2010;24(4):555-63. https:// doi.org/10.1590/S1807-55092010000400012

8. Rocha DC. Relação entre fatores ambientais e a prática de atividade física em idosos participantes do programa nacional marcha e corrida [dissertação]. Porto: Universidade do Porto, 2014. 
9. Tribess S, Junior JSV. Prescrição de exercícios físicos para idosos. Rev Saúde Com. 2005;1(2):163-72.

10. Dias RMR, Gurjão ALD, Marucci MFN. Benefícios do treinamento com pesos para aptidão física de idosos. Acta Fisiatr. 2006;13(2):90-5.

11. Silva WF, Almeida AR, Santos DT, Silveira MB. Os benefícios da atividade física para a qualidade de vida dos idosos em um clube de terceira idade na cidade de Porteirinha, MG. EFDeportes.com [Internet]. 2010 [capturado 2017];15(149). Disponível em: http:// www.efdeportes.com/efd149/os-beneficios-da-atividade-fisicapara-idosos.htm

12. Silva Maitê Fátima da, Goulart Natália Batista Albuquerque, Lanferdini Fábio Juner, Marcon Marcelo, Dias Caroline Pieta. Relação entre os níveis de atividade física e qualidade de vida de idosos sedentários e fisicamente ativos. Rev Bras Geriatr Gerontol. 2012;15(4):634-42. https://doi.org/10.1590/S1809-98232012000400004

13. Mazo Giovana Z, Mota Jorge, Gonçalves Lúcia H.T., Matos Margarida G., Carvalho Joana. Actividade física e qualidade de vida de mulheres idosas da cidade de Florianópolis, Brasil. Rev Port Cien Desp. 2008;8(3):414-23.

14. Alencar Nelyse de Araújo, Souza Júnior José Vitorino de, Aragão Jani Cleria Bezerra, Ferreira Márcia de Assunção, Dantas Estélio. Nível de atividade física, autonomia funcional e qualidade de vida em idosas ativas e sedentárias. Fisioter Mmov. 2010;23(3):473-81. https://doi. org/10.1590/S0103-51502010000300014

15. Fleck MPA, Louzada S, Xavier M, Chachamovich E, Vieira G, Santos L, Pinzon, V. Aplicação da versão em português do instrumento abreviado de avaliação da qualidade de vida "WHOQOL-bref". Rev Saúde Públ. 2000;34(2):178-83. https://doi.org/10.1590/S003489102000000200012

16. IBGE. Sinopse do Censo demográfico [Internet]. 2011 [cited 2017]. Available from: http://www.ibge.gov.br/home/estatistica/populacao/ censo2010/default_sinopse.shtm

17. Silveira MM, Tavares GMS, Zuppa C, Portuguez MW, Silva Filho IG, Carli GA, Pasqualotti A, Colussi EL. Análise da qualidade de vida de idosos frequentadores de oficinas de informática. ConScientiae Saúde. 2014;12:598-603. https://doi.org/10.5585/ConsSaude. v12n4.4327

18. Silveira MM, Pasqualotti A, Colussi EL. Envelhecimento e usuários de informática: repercussões de um programa ergonômico. Estud Interdiscip Envelhec. 2014;19:219-34.

19. Leite MT, Winck MT, Hildebrandt LM, Kirchner RM, Silva LAA. Qualidade de vida e nível cognitivo de pessoas idosas participantes de grupos de convivência. Rev Bras Geriatr Gerontol. 2012;15(3): 481-92. https://doi.org/10.1590/S1809-98232012000300009

20. Busato A, Gallina LS, Téo CRPA, Ferretti F, Pozzagnol M. Autopercepção de saúde e vulnerabilidade em idosos. Rev Baiana Saúde Pública. 2014;38(3):625-35. https://doi.org/10.5327/Z01000233-2014380300010
21. Girardi CE, Heck R, Bobek ML, Benetti ERR, Stumm EMF, Colet CF. Quality of life of people in living groups with diabetes mellitus type 2. J Nurs UFPE. 2015;9(4):7239-46. https://doi.org/10.5205/ reuol.7275-62744-1-SM.0904201508

22. Silva HO, Carvalho MJAD, Lima FEL, Rodrigues LV. Perfil epidemiológico de idosos frequentadores de grupos de convivência no município de Iguatu, Ceará. Rev Bras Geriatr Gerontol. 2011;14(1): 123-33. https://doi.org/10.1590/S180998232011000100013

23. Chaim J, Ramundo ME, Ferreira CAS, Yuaso DR. Prática regular de atividade física e sedentarismo: influência na qualidade de vida de idosas. Rev Bras Ci Env Hum. 2010;7(2):198-209. https://doi. org/10.5335/rbceh.2012.445

24. Cordeiro Juliana, Del Castillo Bruna Lencina, Freitas Caroline Silva de, Gonçalves Marisa Pereira. Efeitos da atividade física na memória declarativa, capacidade funcional e qualidade de vida em idosos. Rev Bras Geriatr Gerontol. 2014;17(3):541-52. https://doi. org/10.1590/1809-9823.2014.13006

25. Vitorino LM, Vianna LAC, Paskulin LMG. Qualidade de vida de idosos em instituição de longa permanência. Rev Latino-Am Enfermagem. 2012;20(6):1-9. https://doi.org/10.1590/S010411692012000600022

26. Silva RS, Silva I, Silva RA, Souza L, Tomasi E. Atividade física e qualidade de vida. Ciênc Saúde Coletiva. 2010;15(1):115-20. https://doi.org/10.1590/S1413-81232010000100017

27. Sanchez MA, Brasil JMM, Ferreira IAM. Benefícios de um programa de atividade física para a melhoria da qualidade de vida de idosos no estado do Rio de Janeiro. RBCEH. 2014;11(3):209-18. https:/ doi.org/10.5335/rbceh.2014.4528

28. Pucci GCMF, Rech CR, Fermino RC, Reis RS. Association between physical activity and quality of life in adults. Rev Saúde Pública. 2012;46(1):1-12. https://doi.org/10.1590/S003489102012000100021

29. Serbim AK, Figueiredo AEPL. Qualidade de vida de idosos em um grupo de convivência. Sci Med. 2011;1(4):166-72.

30. Paskulin LMG, Córdova FP, Costa FM, Vianna LAC. Percepção de pessoas idosas sobre qualidade de vida. Acta Paul Enferm. 2010;23(1):101-7. https://doi.org/10.1590/S0103-21002010 000100016

31. Tavares DMS, Dias FA. Capacidade funcional, morbidades e qualidade de vida de idosos. Texto \& Contexto Enferm. 2012;21(1):112-20. https://doi.org/10.1590/S0104-07072012000100013

32. Benedetti TRB, Mazo GZ, Borges LJ. Condições de saúde e nível de atividade física em idosos participantes e não participantes de grupos de convivência de Florianópolis. Ciênc Saúde Coletiva. 2012;17(8):2087-93. https://doi.org/10.1590/S141381232012000800019 